\title{
Characterization of DECam Focal Plane Detectors
}

H. Thomas Diehl ${ }^{a *}$, Robert Angstadt ${ }^{\mathrm{a}}$, Julia Campa ${ }^{\mathrm{b}}$, Herman Cease ${ }^{\mathrm{a}}$, Greg Derylo ${ }^{\mathrm{a}}$, John H. Emes ${ }^{\mathrm{c}}$, Juan Estrada ${ }^{\mathrm{a}}$, Donna Kubik ${ }^{\mathrm{a}}$, Brenna L. Flaugher ${ }^{\mathrm{a}}$, Steve E. Holland ${ }^{\mathrm{c}}$, Michelle Jonas ${ }^{\mathrm{a}}$, William F. Kolbe $^{\mathrm{c}}$, John Krider ${ }^{\mathrm{a}}$, S. Kuhlmann ${ }^{\mathrm{d}}$, Kevin Kuk ${ }^{\mathrm{a}}$, Marino Maiorino ${ }^{\mathrm{b}}$, Nick Palaio ${ }^{\mathrm{c}}$, Andres Plazas ${ }^{\mathrm{e}}$, Natalie A. Roe ${ }^{c}$, Vic Scarpine ${ }^{a}$, Ken Schultz ${ }^{a}$, Terri Shaw ${ }^{a}$, Hal Spinka ${ }^{d}$, Walter Stuermer ${ }^{a}$

${ }^{\mathrm{a}}$ Fermi National Accelerator Laboratory, Batavia, IL USA 60510; ${ }^{\mathrm{b}} \mathrm{Ctr}$. de Investigaciones Energeticas, Medioambientales y Tecnologicas, Madrid, Spain; ${ }^{\mathrm{c}}$ Lawrence Berkeley National Laboratory, Berkeley, CA USA 94720, ${ }^{\mathrm{d}}$ Argonne National Laboratory, Argonne IL USA 60439, ${ }^{e}$ Dept.of Physics and Astronomy, University of Pennsylvania, 209 S. $33^{\text {rd }}$ St., Philadelphia, PA USA 19104-6396

\section{FOR THE DARK ENERGY SURVEY COLLABORATION}

\begin{abstract}
DECam is a 520 Mpix, 3 square-deg FOV imager being built for the Blanco $4 \mathrm{~m}$ Telescope at CTIO. This facility instrument will be used for the "Dark Energy Survey" of the southern galactic cap. DECam has chosen $250 \mu \mathrm{m}$ thick CCDs, developed at LBNL, with good QE in the near IR for the focal plane. In this work we present the characterization of these detectors done by the DES team, and compare it to the DECam technical requirements. The results demonstrate that the detectors satisfy the needs for instrument.
\end{abstract}

Keywords: CCDs, Mosaic camera, Dark Energy Survey

\section{INTRODUCTION}

The Dark Energy Survey [1-2] (DES) will measure dark energy parameters using four complementary techniques: weak gravitational lensing, galaxy cluster counting, baryon acoustic oscillations, and supernovae. The data will be collected during a 5000 square degree survey of the southern galactic cap and a smaller repeated supernovae survey during 525 nights of observing from 2011 to 2016. The survey requires a new instrument, the Dark Energy Camera (DECam), to be built and installed in the prime focus of the 4-meter Blanco telescope at Cerro Tololo in Chile.

The DECam [3] has a 3 square-degree field of view accomplished using a new optical corrector with one of the largest lenses ever produced for optical astrophysics, and an 8-filter housing that will contain g,r,i,z, and Y filters. The imager is a 520 Mpixel digital camera comprised of sixty-two 2048 x 4096 CCDs. An additional twelve 2048 x 2048 CCDs will be used for guide and focus applications. The CCD readout is performed using a system [4] based on the National Optical Astronomy Observatory (NOAO) Monsoon electronics.

The CCDs are 250-micron thick, back-illuminated, red-sensitive devices [5-6] designed by Lawrence Berkeley National Laboratory (LBNL) and produced by a combination of Dalsa and LBNL. The CCD is back-illuminated through an antireflection coating and is fully-depleted by application of bias voltage, typically 40 volts, to a thin backside conductive layer. Photons create holes in the thick substrate, which is n-type silicon, that are collected in a p-type channel. High quantum efficiency in the near-infrared wavelengths results because the CCDs are thicker than traditional thin astronomical CCDs. Like conventional CCDs, the LBNL devices use three "vertical" clocks to transfer rows of charge to the serial register, and three "horizontal" clocks to transfer individual pixels from the serial register to the output amplifier. The pixel geometry is 15 -micron squares.

\footnotetext{
*Diehl@FNAL.GOV; phone 1-630-840-8307
} 
This paper will present the CCD technical specifications, the plan for characterizing the CCDs and their packages, and the test results obtained thus far.

\subsection{CCD Production and Packaging}

The CCDs are produced by DALSA and LBNL in 24-wafer "Lots" with four 2048 x 4096 pixel CCDs, one 2048 x 2048 pixel CCD, and four 1024 x 512 pixel CCDs on each wafer. The processing starts at DALSA. Three wafers per lot are finished at DALSA and are 650 microns thick, too thick to be fully depleted at the desired substrate voltage. The remaining 21 wafers are sent to LBNL for thinning to 250 microns and for the backside-processing steps that complete the CCD production. LBNL ships the CCDs to the Fermi National Accelerator Laboratory (Fermilab), along with results from readout tests of each $2 \mathrm{k} \times 4 \mathrm{k}$ CCD. This test data is accumulated using a cold probe station operating at temperature $-45 \mathrm{C}$. Defects are identified and classified automatically using data taken at different clock and substrate bias voltage levels. Though that is warmer then the expected operating temperature of $-100 \mathrm{C}$, this data is vital for determining those devices that are potentially of high quality and, therefore, suitable for packaging and testing.

The CCDs are mounted on packages [7] at Fermilab. We use two kinds of packages. The "Picture Frame" package [8] is an easily-manufactured assembly useful for CCD tests. For a "Picture frame" package, the CCD is glued to a stiff aluminum-nitride blank substrate and then wire-bonded to a small g-10 printed circuit board. However, it is not a 4-sidebuttable design, which is required for a mosaic camera such as DECam. The 4-side-buttable package is called a "Pedestal Package". The CCD is held flat while it is glued to an assembly comprised of an aluminum nitride circuit board, aluminum nitride spacer, and flat Au-plated INVAR foot. The CCD is wirebonded to the aluminum nitride circuit. The aluminum nitride circuit has an Airborn 37-pin N-series two-row female connector for connection to the CCD readout electronics.

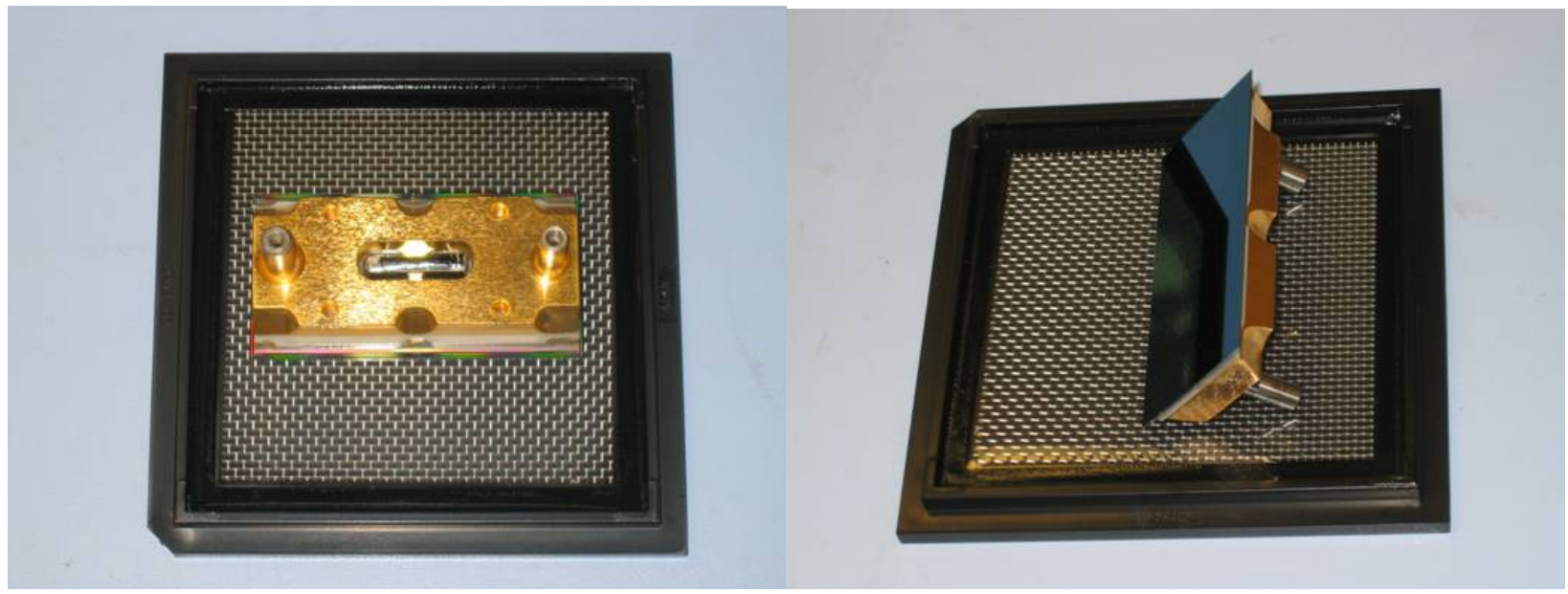

Figure 1-1 A 4-side-buttable pedestal package is displayed. View of underside of a pedestal package sitting on a gel-pack showing the Au-plated INVAR foot, two alignment pins, the connector, the edge of the AIN blank with cut-outs for the wirebonds and the edge of the CCD (left). The same CCD viewed in perspective showing the front side of the CCD, which is dark because of the anti-reflective coating (right). The cut-outs in the Au-plated INVAR foot allow the wirebonding machine viewing access to the AlN circuit and CCD bond pads.

\section{TECHNICAL SPECIFICATIONS}

\subsection{Typical Characteristics of the LBNL CCD and DECam CCD Technical Requirements}

Technical specifications for the CCDs were determined from the Dark Energy Survey science requirements. These are listed in Table Table 2-1 along with the typical characteristics of the LBNL fully-depleted, red-sensitive CCD. In particular, these CCDs have high quantum efficiency in the wavelength range 800-1050 nanometers. Because they are 250 microns thick, fringing is expected to be minimal in the $\mathrm{f} / 3$ beam of the Blanco 4-meter primary mirror. The CCDs are required to be readout in 17 seconds, the telescope slewing time during the survey. That readout rate corresponds to $250 \mathrm{k} \mathrm{pixel} / \mathrm{second}$ at $<15 \mathrm{e}^{-}$noise. The typical observing is expected to produce a point-spread-function (PSF) of 0.9 ”. 
The technical requirement is that the instrument delivers a PSF $<0.55$ " resulting in a budget for charge diffusion of 7.5 microns, corresponding to 0.14 ". The CCDs are required to be flat, uniformly thick, and form a focal plane with a peakto-peak variation of less than 30 microns; the individual CCD flatness requirements are discussed in Section 4.1. The CCDs are required to be of high quality; the mean percentage of pixels that are out-of-tolerance will be $<0.5 \%$ with no single device having more than $2.5 \%$. The definition of cosmetic defects and the allowed tolerances are described in detail in Section 4.3.

Table 2-1 DECam CCD technical requirements and the typical characteristics of the LBNL fully-depleted CCD.

\begin{tabular}{|c|c|c|}
\hline & $\begin{array}{l}\text { LBNL CCD } \\
\text { Performance }\end{array}$ & DECam Requirements \\
\hline Pixel array & 2048 x 4096 pixels & 2048 x 4096 pixels \\
\hline Pixel size & $15 \mu \mathrm{m} \times 15 \mu \mathrm{m}$ & $15 \mu \mathrm{m} \times 15 \mu \mathrm{m}$ \\
\hline \# Outputs & 2 & 2 \\
\hline QE(g,r,i,z) & $70 \%, 90 \%, 90 \%, 75 \%$ & $60 \%, 75 \%, 60 \%, 65 \%$ \\
\hline QE Instability & Stable & $<0.3 \%$ in $12-18 \mathrm{hrs}$ \\
\hline $\begin{array}{l}\text { QE Uniformity in focal } \\
\text { plane }\end{array}$ & uniform & $<5 \%$ in $12-18 \mathrm{hrs}$ \\
\hline Full well capacity & $170,000 \mathrm{e}^{-}$ & $>130,000 \mathrm{e}^{-}$ \\
\hline Dark current & $2 \mathrm{e}^{-} / \mathrm{hr} / \mathrm{pixel}$ at $120^{\circ} \mathrm{K}$ & $<\sim 25 \mathrm{e}^{-/ h r} /$ pixel \\
\hline Persistence & Erase mechanism & Erase mechanism \\
\hline Read noise & $7 \mathrm{e}^{-} @ 250 \mathrm{kpixel} / \mathrm{s}$ & $<15 \mathrm{e}^{-} @ 250 \mathrm{kpix} / \mathrm{s}$ \\
\hline $\begin{array}{l}\text { Charge Transfer } \\
\text { Inefficiency }\end{array}$ & $<10^{-6}$ & $<10^{-5}$ \\
\hline Charge diffusion & 6-7 $\mu \mathrm{m}$ & $1 \mathrm{D} \sigma<7.5 \mu \mathrm{m}$ \\
\hline Cosmetic Requirements & & $\begin{array}{c}<\# \text { Bad pixels }> \\
<0.5 \%\end{array}$ \\
\hline Linearity & Better than $1 \%$ & $1 \%$ \\
\hline Package Flatness & $<10 \mu \mathrm{m}$ & See Section 4.1 \\
\hline
\end{tabular}

\section{CCD TESTING INFRASTRUCTURE AND TEST STANDS}

CCD packaging and testing is carried out in the Silicon Detector Facility (SiDet) at Fermilab. This facility is well-known for design, assembly, and testing of silicon strip and pixel vertex detectors for use in elementary particle physics experiments at Fermilab and at CERN. SiDet has numerous clean areas, wirebonding machines, optical and mechanical measurement systems, and probe systems, as well as the infrastructure required to work with and protect the electrostatic sensitive CCDs from inadvertent damage. Finally, SiDet has the skilled engineers and technicians required to perform this kind of work.

\subsection{CCD Test Stand}

The CCDs are tested using the optical setup shown in Figure 3-1. A single CCD is housed in a thermally-controlled vacuum dewar that is cooled to operating temperature $(-100 \mathrm{C})$ using LN2. A standard set of optical equipment allows uniform or patterned illumination of the CCD surface in the desired wavelength. Exposure times are controlled by a small shutter. The optical path is light-tight. The shutter, filter wheel, and monochrometer are controlled using GPIB through a TCL interface. All CCD test sequences use automated imaging scripts. The vacuum dewar also contains an ${ }^{55} \mathrm{Fe}$ radioactive source housed on an arm that can be rotated to a location in front of the CCD. The CCDs are readout using variants of NOAO Monsoon. The data is organized as a standard FITS image. This test stand has been replicated four times so that we can test CCDs at the rate we package them during production. 


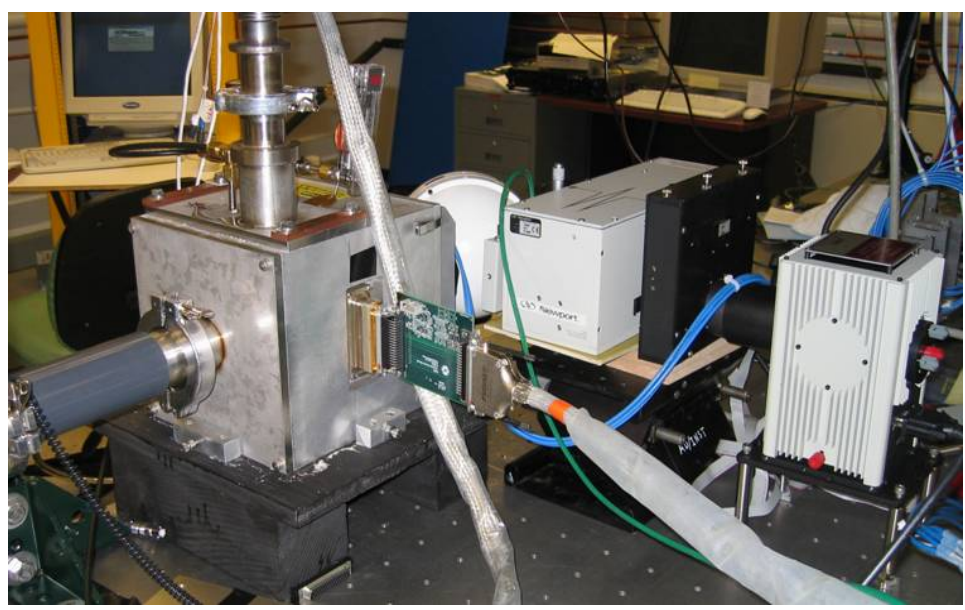

Figure 3-1 Typical CCD optical test stand. From the right, the light source is a tungsten arc lamp, filter changer, monochrometer, shutter, integrating sphere. The light enters the vacuum dewar through a fused-silica window (facing away). The CCD package is mounted on a cold-plate that faces the window. It is cooled with liquid nitrogen. The operating temperature is maintained using a heater powered by a LakeShore temperature controller. A preamp is visible on the right side of the dewar as well as the cable bundle to the Monsoon electronics.

\section{CCD TEST RESULTS}

From August 2005 to April 2008 we characterized a sample of engineering grade 2k x 4k and ungraded 2k x 2k LBNL CCDs to make sure they satisfied all technical specifications. During the same period, we developed a streamlined, automated test procedure. We report the results ${ }^{1}$ in this section.

\subsection{CCD Thickness and Flatness}

The individual pedestal package CCDs are required to be uniformly thick and flat. The flatness specification originates from the requirement that we be able to measure astrometric corrections for weak-lensing analysis. The requirement is two-fold. First, detector flatness on a $1 \mathrm{~cm}^{2}$ scale is $<3$ microns. Second, detector flatness between adjacent $1 \mathrm{~cm}^{2}$ regions on a given $\mathrm{CCD}$ must be $<10$ microns.

The package thickness is measured using a coordinate measurement machine equipped with a microscope camera. Using camera focus to inspect heights, a grid of 15 elevation points is measured on the exposed surface of the CCD relative to the flat fixture on which it is mounted. The repeatability of the autofocus feature of the camera is \pm 1 micron. The package thickness is controlled by the assembly hardware and we have found that the thickness is a repeatable feature of the assembly process. We have measured the thickness of two packages made using mechanical grade CCDs. They were uniformly thick to within about 4 microns.

The CCD flatness is measured at operating temperature through the dewar window. This is accomplished using a confocal chromatic displacement measurement system from Micro-Epsilon Corp. This device focuses light of different frequencies at different distances from the instrument. Light that is reflected back from the CCD surface is directed through a pinhole camera and diffraction grating. The color of light that is brightest is that focused on the CCD surface. The advertised accuracy of the device is 7 microns over a $24 \mathrm{~mm}$ range. The working distance is centered at $250 \mathrm{~mm}$ from the imager head. This imager is mounted on a set of straight motorized stages from Physik Instrumente in $\mathrm{x}-\mathrm{y}$ configuration. They have a straightness of 1 micron per $100 \mathrm{~mm}$ travel, suitable for the flatness measurement. The system is set on a granite block with a large thermal mass so as to avoid systematic uncertainties from vibration and thermal expansion or contraction of the measurement system.

The distance to the CCD is scanned in a $63 \times 123$ point grid resulting in measurement of the full CCD surface with 0.5 $\mathrm{mm}$ between grid points. The scan is performed 3 to 5 times. For each scan a best-fit-plane is subtracted from the data. The median of the resulting flatness measurement is then calculated at each grid point. The results are averaged in $1 / 4 \mathrm{~cm}$

\footnotetext{
${ }^{1}$ Unless specified otherwise, all results are from CCD temperature $=-100 \mathrm{C}$ and at $250 \mathrm{kpixels} / \mathrm{second}$ readout speed.
} 
and $1 \mathrm{~cm}$ squares. Figure 4-1 shows a "topographical map" of the surface of one of the CCDs. It passes the two flatness specifications. The mean rms difference of $1 / 4 \mathrm{~cm}^{2}$ regions from the parent $1 \mathrm{~cm}^{2}$ regions is 1.2 microns. The maximum rms deviation is 2.27 microns (in the lower left corner of the figure). The average height difference between adjacent 1 $\mathrm{cm}$ squares is 1.75 microns. The maximum difference is 8.32 microns (near the upper right corner of the figure).

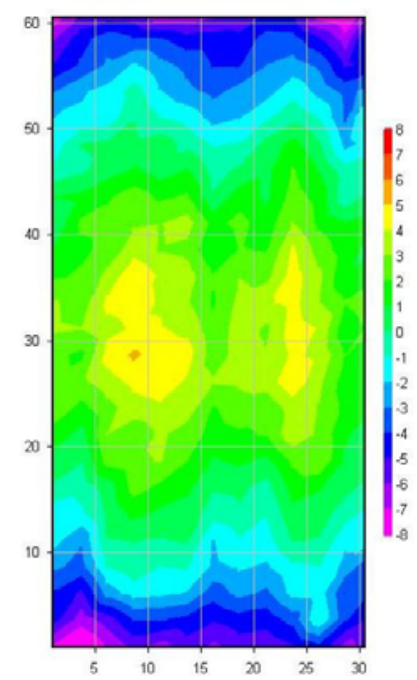

Figure 4-1 Flatness scan of V2.1 CCD package S2-21. The $\mathrm{x}$ and $\mathrm{y}$ axes are units $\mathrm{mm}$. The color represents the distance in microns from the best fit plane of the flatness measurements. The full scale is \pm 8 microns.

\subsection{CCD Linearity, Full Well, and Persistance}

Linearity and CCD well-depth are determined from the photon yield curve and the photon transfer curve. The photon yield curve shows the number of ADC counts versus exposure time using a flat-field white-light source.

Figure 4-2 shows the photon yield curve for one CCD and the relative residuals from a linear fit. With residuals $<0.2 \%$, the CCD passes the requirements. The photon transfer curve is a set of images taken with first increasing and then decreasing light levels, two images at each light level. Assuming Poisson statistics for the number of electrons per pixel, the gain and deviation from linearity can be obtained. The variance is plotted against the mean number of photons. Figure 4-2 also shows the photon transfer curve for one CCD.
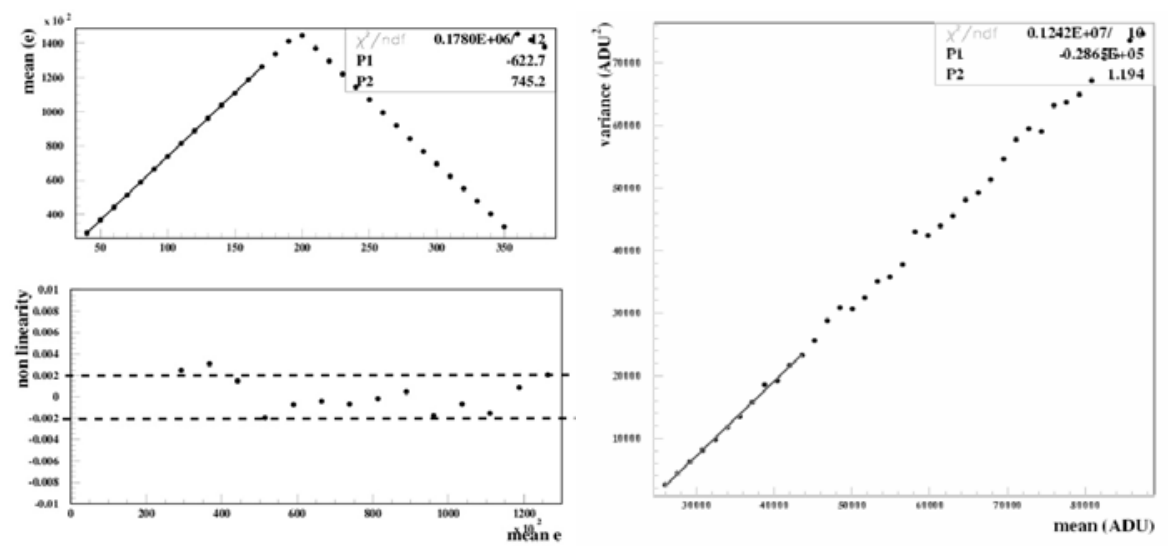

Figure 4-2 Top Left) Light level measured for the linearity test in a $2 \mathrm{k}$ x $2 \mathrm{k}$ device from production Lot $2 \mathrm{~A}$. A set of exposures is taken with increasing and then decreasing exposure time. The $\mathrm{x}$-axis shows the time sequence (seconds) of the exposures. Bottom Left) The non-linearity measured for the data in the top panel as a function of mean signal. The plot shows that less than $0.2 \%$ nonlinearity is achieved up to 120,000 electrons. Note that the $\mathrm{x}$-axis in the bottom plot is in unit 100's of electrons. Right) Photon transfer curve. Photon Transfer Curve for a 250 micron, backside illuminated DES CCD. The photon transfer curve shows the variance as a function of the mean, obtained by taking a series of exposures of increasing time length. The slope of this curve is the gain, in units electrons per ADU. 
Residual image (persistence) is charge left on the pixels from previous exposures. It is usually most evident on dark areas that previously were at or near saturation. In order to eliminate residual image from these devices, the CCD is erased. The procedure is to lower the substrate voltage from $40 \mathrm{~V}$ to $0 \mathrm{~V}$, invert the vertical clocks, and then raise the substrate voltage. Using this procedure, the residual image is negligible, even after recording an image with pixels illuminated to many times the full-well level. The photon yield curve and photon transfer curve also supply data that allows us to determine the fraction of the CCD that passes the cosmetic requirements.

\subsection{Cosmetic Requirements}

We have performed detailed studies of cosmetic defects in our devices. The cosmetic requirements are checked taking long dark exposures, flat exposures at different light levels and by doing charge pumping to detect traps. About 2.5 hours are spent on each detector to do these measurements. Table 4-1 lists the categories of cosmetic defects and their descriptions.

In addition to the imperfect pixels, there will be a loss of $10 \%$ of the focal plane area to gaps between the individual CCDs. Another $1 \%$ is lost to edge effects, defined as the area of a detector in which the effective size of a pixel is more than $10 \%$ different from the neighbor pixel, as well. We therefore have a requirement that the average fraction of unusable pixels in the focal plane should be less than $0.5 \%$. This requirement is then set on the average for the whole set of CCDs for the DES focal plane. In addition, data processing experience suggests that no $1 / 2$ section of any CCD on the focal plane be more than $2.5 \%$ unusable, so we set this requirement on each CCD.

Table 4-1 Categories of cosmetic defects and their descriptions.

\begin{tabular}{|c|c|}
\hline Cosmetic Defect & Description \\
\hline 1. Dark Pixels & $\mathrm{QE}<20 \%$ below the flat field median. \\
\hline 2. Hot Pixels & $\begin{array}{l}\text { Dark current larger than } 6300 \text { electrons per hour at the } \\
\text { nominal operating temperature. }\end{array}$ \\
\hline 3. Traps & Deeper than 700 electrons. \\
\hline 4. Non-linearity & $\begin{array}{l}\text { Response }>1 \% \text { different at roughly } 50 \% \text { and } 80 \% \text { of the } \\
\text { detector full well. }\end{array}$ \\
\hline 5. Instability & $\begin{array}{l}\text { Pixels that change response, dark current, or linearity by } \\
\text { more than } 10 \% \text { over } \sim 30 \text { minute timescales. }\end{array}$ \\
\hline
\end{tabular}

Because we have reserved the best $2 \mathrm{k} x$ 4 $\mathrm{k}$ CCDs for production, which we haven't started as of this time, we don't have final cosmetic results from $2 \mathrm{k} \times 4 \mathrm{k}$ thinned CCDs. Instead, since we don't have cold probe data for the $2 \mathrm{k} \times 2 \mathrm{k}$ detectors, and, therefore, there is no way to reserve the best of these for production, we performed the complete cosmetic analysis on four $2 \mathrm{k} \times 2 \mathrm{k}$ DECam CCDs. The fraction of bad pixels measured these detectors was: $0.014 \%, 0.011 \%$, $0.012 \%$, and $0.38 \%$. All 4 detectors satisfy the requirement of $<2.5 \%$ unusable area per CCD, and as a set they are below the $0.5 \%$ average required for the focal plane. The best 3 detectors in this sample would be qualified as grade 0 (the best grade) according to the E2V standard [9] for grading cosmetic defects on scientific CCDs.

\subsection{Charge Transfer Efficiency}

The charge transfer efficiency is determined by both the extended-pixel-edge-response (EPER) method, and by the use of an ${ }^{55} \mathrm{Fe}$ source. In the EPER method the CCD is illuminated with a light source and the fraction of charge transferred from active pixels into the overscan region provides the charge transfer inefficiency (CTI). The CTI, in practice, depends on the levels of various CCD clocks. Each of the four clock rails (horizontal upper and lower rail, vertical upper and lower rail) used for the charge transfer between pixels is varied between $0.5 \mathrm{~V}$ and $10 \mathrm{~V}$ in steps of $0.5 \mathrm{~V}$. A 10 second exposure is taken for each voltage level. So far we have found a single set of clock voltages for which all the (otherwise functional) CCDs pass the CTI technical requirement for both horizontal and vertical clocks. The CTI for a typical device is $10^{-7}$.

The other method for measuring CTI is based on the illumination of the detectors with X-rays. We illuminate the CCD with $\mathrm{x}$-rays from a ${ }^{55} \mathrm{Fe}$ source. The most prominent feature in the $\mathrm{x}$-ray energy spectrum is $5.9 \mathrm{keV}$ and for most $\mathrm{x}$-ray hits all of the energy is deposited into one pixel. The ratio of measured charge with respect to expected as a function of 
pixel position in the detector charge provides the CTI. Because of diffusion in our $250 \mu \mathrm{m}$-thick back-illuminated CCDs, the charge is spread out. However, we have measured the CTI in some packages built with the front-side exposed. The measured CTI is $6 \times 10^{-6}$ for the vertical clocks and $3 \times 10^{-6}$ for the horizontal clocks.

\subsection{Read Noise}

The readout noise depends on the readout speed. We test the CCDs at our nominal operating readout speed of $4 \mu$ s per pixel. After measuring the gain with the photon transfer curve or with ${ }^{55} \mathrm{Fe} X$-rays, we can convert the readout noise from analog-to-digital units (ADU) to electrons. At present we achieve 8 electrons readout noise at $3.7 \mu$ s per pixel, a little better than the specifications on both accounts. To accomplish this requires a source-follower circuit mounted in close proximity to the CCD. The result satisfies the technical requirements for readout speed and readout noise. Incidentally, when we read out as slowly as $30 \mu \mathrm{s} /$ pixel, we achieve $<2$ electrons noise.

\subsection{Quantum Efficiency}

The long wavelength response of the LBNL CCDs makes this survey possible. We measure the absolute quantum efficiency $(\mathrm{QE})$ as a function of wavelength. Care is taken to understand and monitor the light power incident on the surface of the CCDs. This is done using two calibrated photodiodes, one that monitors the source power and the other that monitors the power that arrives at the CCD. Figure 4-3 shows the absolute and relative quantum efficiency (QE) for two DES CCDs, as measured at Fermilab, in comparison with measurements of a similar 250-micron thick, backside illuminated CCD at LBNL. We see that the key feature, that of high quantum efficiency at $1000 \mathrm{~nm}$, is evident and that the results are in qualitative agreement at all wavelengths. The uncertainty in our absolute QE measurements is $3 \%$ at all wavelengths. The uncertainty in relative $\mathrm{QE}$ is negligible. We see that the $\mathrm{QE}$ passes the technical requirements.

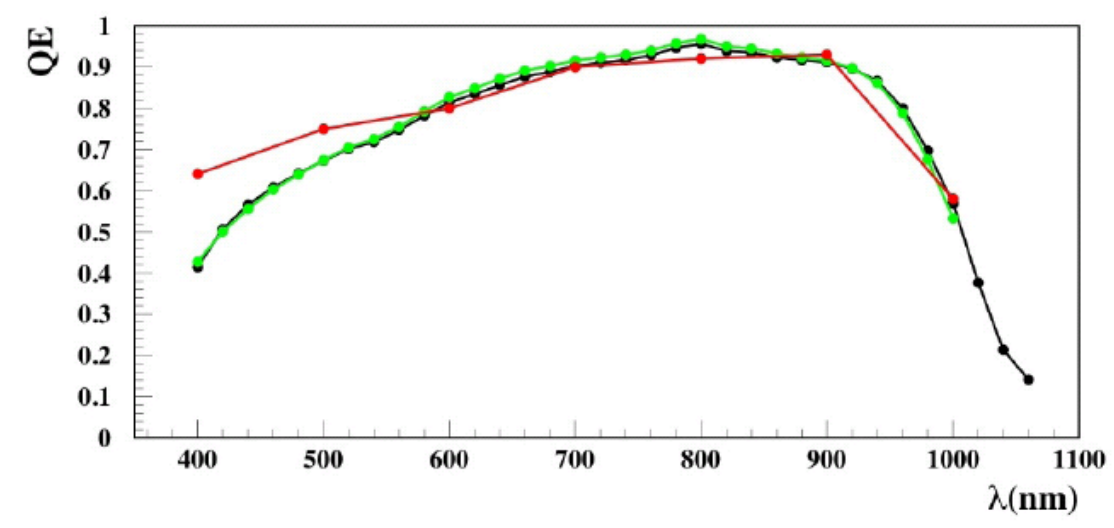

Figure 4-3 Absolute Quantum Efficiency vs wavelength for DECam CCDs. The absolute QE for two different $2 \mathrm{k} x 2 \mathrm{k}$ CCDs from Lot $2 \mathrm{~A}$ (green and black) compared with the results in a engineering CCD at LBNL (red). The CCD temperature is $-100 \mathrm{C}$. In black and white print, the green curve appears as grey and the red curve is the one with higher $\mathrm{QE}$ at $400 \mathrm{~nm}$.

\subsection{Quantum Efficiency vs. Temperature}

The QE must be stable to within $0.3 \%$ over a $12-18$ hour time span. The QE of the red end of the spectrum is sensitive to the CCD temperature. We measured that at four temperatures over the full range of wavelengths using the monochrometer. Figure 4-4 shows the result. It is very wavelength dependent. The plots show a $+4 \% \mathrm{QE}$ change at 1000 $\mathrm{nm}$ when the temperature is changed by $10 \mathrm{~K}$. This has been found to be device independent. This indicates that to achieve technical requirement we need temperature stability on the focal plane of̣ $\pm 0.25 \mathrm{~K}$.

Technical requirements demand the absolute QE of the detectors in the focal plane must be uniform to within 5\%. The current plan does not include a selection of the CCDs to accommodate a temperature gradient on the focal plane to achieve this requirement. Considering the results presented here, this requirement means that the temperature of the focal plane has to be uniform to $10 \mathrm{~K}$. 

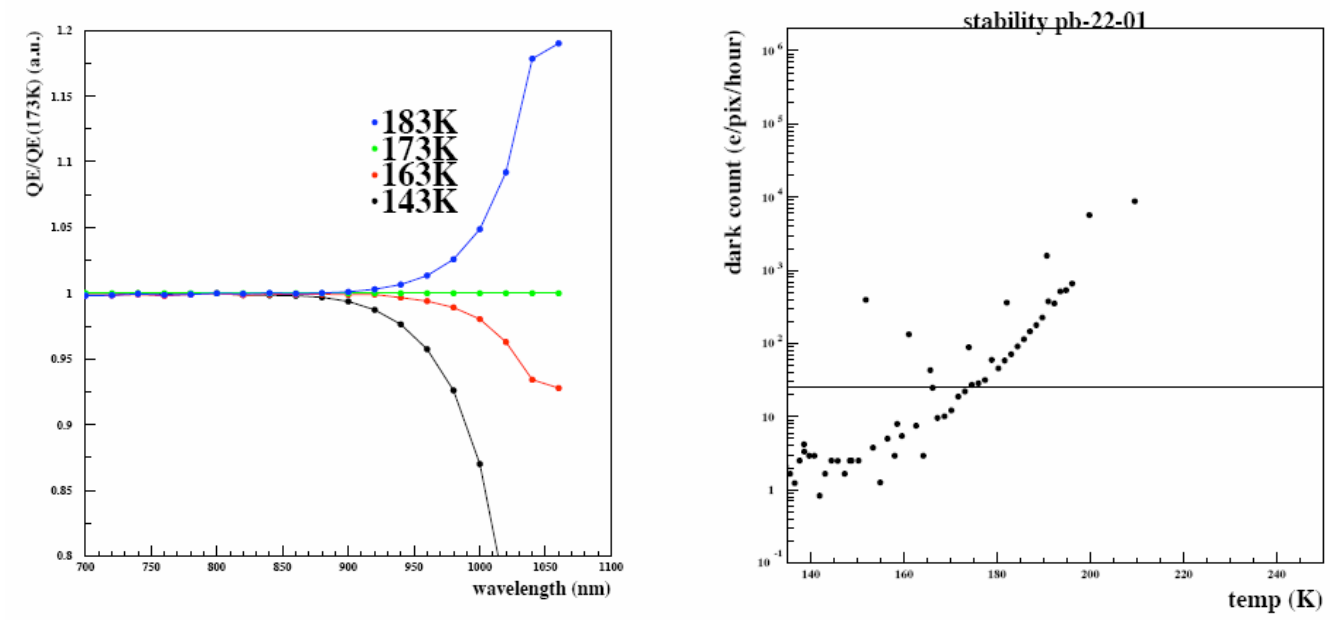

Figure 4-4 QE relative to operating temperature $(173 \mathrm{~K})$ as a function of temperature and wavelength (left). Note the full scale represents a $20 \%$ change in the QE. Dark current as a function of temperature (right).

\subsection{Dark Current}

The dark current specification for the DECam detectors is 25 electrons per pixel per hour. The measurements of dark current for an engineering DECam detector are shown in Figure 4-4. The results indicate that the technical specification for DECam is achieved below $180 \mathrm{~K}$. In order to select an operating temperature for the CCDs, we also studied the QE as a function of temperature, as previously described. A trade-off between increased QE at higher temperatures and decreased dark current at lower temperatures, including expected sky background was performed. This same trade-off study included the trade-off on the CCD thickness. Since the QE for wavelengths around $1000 \mathrm{~nm}$ drops significantly when the temperature is reduced, we take $173 \mathrm{~K}$ as our operating point.

\subsection{Charge Diffusion}

We measure charge diffusion using two techniques. We developed a new technique [10] to measure charge diffusion based on Fourier analysis of the spatial resolution of a double slit diffraction pattern as the substrate voltage is varied. We measured the diffusion vs. substrate voltage in several $2 \mathrm{k}$ x $2 \mathrm{k}$ CCDs. The results obtained indicate that for Vsub $=$ $40 \mathrm{~V}$, the tested DES CCDs have a dispersion due to charge diffusion $\sigma$ (diffusion) $<7.5 \mu \mathrm{m}$. The technique used simplifies significantly the setup needed for the measurement with respect to the more traditional "spot machine" method [11] and permits the characterization of the diffusion in a large number of the DECam CCDs. However, the new technique is not capable of making a localized measurement of diffusion in a very small area of the sensor. For that we use $\mathrm{x}$-rays from ${ }^{55} \mathrm{FE}$. Figure 4-5 shows the amount of diffusion vs. substrate voltage $\left(\mathrm{V}_{\text {sub }}\right)$ from the $\mathrm{x}$-ray technique.

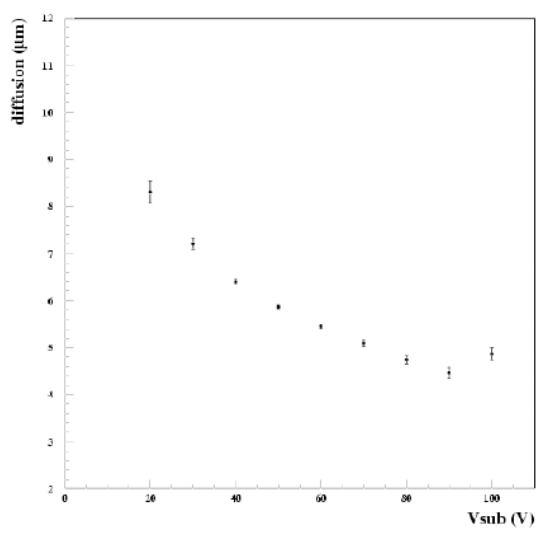

Figure 4-5 Charge diffusion vs. $\mathrm{V}_{\text {sub }}$ in a 250 micron thick DECam CCD as measured using x-ray hits from ${ }^{55} \mathrm{Fe}$. The operating point for $\mathrm{V}_{\text {sub }}$ is $40 \mathrm{~V}$, corresponding to $\sigma$ (diffusion $)=6.5$ microns. 


\subsection{CCD Testing Sequence}

At the peak of production rate we will test four CCDs per week. The tests are performed in three "stages" with better devices undergoing all tests and lower quality devices rejected as soon as possible. We rely heavily on the automated scripts to control the optical equipment and perform the imaging sequences required for the tests.

Stage 1 testing is performed on every packaged device. These tests are completed during the first day or night of testing. These include: a photon transfer curve; a clock rail scan to verify the CTI, noise dependence and defect dependence on the clock level; output gate transfer, a test which allows to determine when channel potential under the output gate - and thus, whether or not the bias voltages are sensible; a charge-pumping test to detect charge traps; and a series of dark and flat exposures to test for cosmetic defects. The Stage 1 test data is analyzed by physicists at FNAL and at our collaborating institutions. Devices passing Stage 1 testing will remain cold for further studies. We expect at least $3 / 4$ of the CCDs will pass the Stage 1 tests.

Stage 2 testing will require approximately 2 more days to complete. These tests are carried out in the same "Cube" as the Stage 1 tests; thus they don't require warming up the CCD, nor transfer to another test stand. The tests include the quantum efficiency, charge diffusion, and $\mathrm{QE}$ and noise vs. temperature measurements. We expect most devices that passed the Stage 1 tests will also pass the Stage 2 tests.

Stage 3 testing is for package flatness; it requires several days to perform as well as transfer to a new test setup. Only a fraction of the devices will be tested for flatness, which we have found is a reliable property determined by the CCD assembly jigs.

A database for storing CCD testing results has been produced. It includes fields for all the technical requirements and operating voltages. It has easy data-entry and sort-able and searchable fields.

We have tested the throughput of our CCD test procedure. We have demonstrated the capability to process five CCDs per week through Stage 1 and Stage 2 testing, as an exercise for the facility. This rate will allow us to meet the production schedule. Also, we have produced a Monte Carlo (queuing) simulation that allows us to predict the number of devices that are being tested simultaneously at any given stage. The same simulation shows we will be able to keep up with CCD production in the event that one of the test "Cubes" drops out of operation for a short time period.

\section{SYSTEM TESTS}

\subsection{System Tests}

We perform electronic and mechanical systems tests in a full-sized prototype camera called the "Multi CCD Test Vessel" or MCCDTV, for short. Figure 5-1 shows a photograph of the front of the dewar, including 4 2k x 4k V1 CCD packages mounted on the first version of the prototype support plate. The camera has a 2"-thick fused-silica window. We have designed and built a light-tight baffle system so that we can illuminate CCDs through the window. It is used for developing the software that controls readout of multiple CCDs in a single image, for system tests of the electronics, for development of focal plane support plate design [12], and for development of the cooling [13] and control systems. We describe a few results here.

We have demonstrated the readout of 4 CCDs in the MCCDTV at same noise and readout speed as in the "Cubes". Tests with six CCDs are underway at this time.

The CCD crosstalk has been carefully studied for single and multiple CCDs. The measured crosstalk between the two readout channels was $X(C C D)=2.7 \times 10^{-4}$ for readout speed $3.7 \mu$ s per pixel when the $C C D$ was read out from a single CCD dewar (Cube). In the MCCDTV, the crosstalk for two channels of the same CCD was $X(C C D)<1 \times 10^{-5}$. Between two CCDs in the multi-CCD dewar the crosstalk was $\mathrm{X}(\mathrm{MCCDTV})=1.4 \times 10^{-4}$. These crosstalk measurements include the contribution from CCD packaging, cables, and interface boards inside the various dewars. The results show that the crosstalk satisfies the requirement that $\mathrm{X}($ total $)<10^{-3}$. 


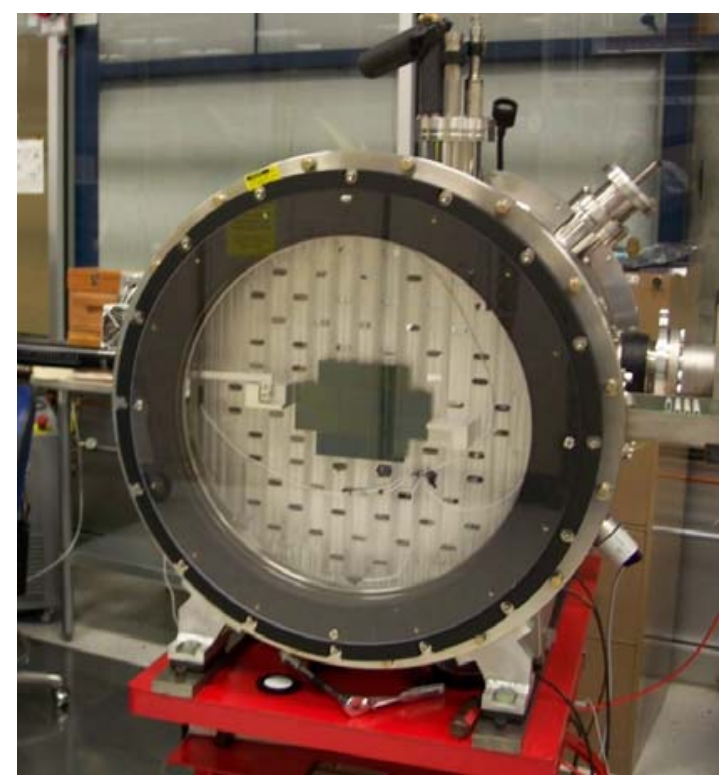

Figure 5-1 The Multi-CCD Test Vessel with 10 2kx4k CCDs mounted on the prototype focal plane support plate.

\section{DISCUSSION AND SUMMARY}

DECam is a 520 Mpix, 3 square-deg FOV imager being built for the Blanco 4m Telescope at CTIO. This facility instrument will be used for the "Dark Energy Survey" of the southern galactic cap. DECam has chosen $250 \mu \mathrm{m}$ thick CCDs, developed at LBNL, with good QE in the near IR for the focal plane. In this work we presented the characterization of these detectors done by the DES team. We described the test equipment, test procedure, and results. The results demonstrate that the detectors satisfy the needs for instrument. In addition we showed results from a few systems tests performed with a prototype multi-CCD focal plane and test vessel.

The Dark Energy Camera is expected to be completed and brought to CTIO in late 2010. Most systems will have been commissioned at Fermilab so that final assembly and commissioning on the dome floor will be accomplished quickly. DECam is expected to be ready to mount on the Blanco Telescope early in 2011.

\section{ACKNOWLEDGEMENTS}

Funding for the DES Projects has been provided by the U.S. Department of Energy, the U.S. National Science Foundation, the Ministry of Science and Education of Spain, the Science and Technology Facilities Council of the United Kingdom, the Higher Education Funding Council for England, the National Center for Supercomputing Applications at the University of Illinois at Urbana-Champaign, the Kavli Institute of Cosmological Physics at the University of Chicago, Financiadora de Estudos e Projetos, Fundação Carlos Chagas Filho de Amparo à Pesquisa do Estado do Rio de Janeiro , Conselho Nacional de Desenvolvimento Científico e Tecnológico and the Ministério da Ciência e Tecnologia and the Collaborating Institutions in the Dark Energy Survey.

The Collaborating Institutions are Argonne National Laboratories, the University of Cambridge, Centro de Investigaciones Energeticas, Medioambientales y Tecnologicas-Madrid, the University of Chicago, University College London, DES-Brazil, Fermilab, the University of Edinburgh, the University of Illinois at Urbana-Champaign, the Institut de Ciencies de l'Espai (IEEC/CSIC), the Institut de Fisica d'Altes Energies, the Lawrence Berkeley National Laboratory, the University of Michigan, the National Optical Astronomy Observatory, the Ohio State University, the University of Pennsylvania, the University of Portsmouth and the University of Sussex. 


\section{REFERENCES}

[1] T. Abbott et al., "The Dark Energy Survey”, astro-ph/0510346.

[2] B. Flaugher, "The Dark Energy Survey", Int. J. Modern. Phys. A20, 3121 (2005).

[3] D. L. Depoy et al.,"The Dark Energy Camera (DECam)", these proceedings.

[4] Miquel Barcelo et al., "Front-end electronics for the Dark Energy Camera (DECam)", these proceedings.

[5] S. E. Holland, D. E. Groom, N. P. Palaio, R. J. Stover, and M. Wei, IEEE Trans. Electron Dev. 50, 225 (2003).

[6] N. A. Roe et al., "Radiation-tolerant, red-sensitive CCDs for dark energy investigations", Nucl. Inst. And Meth. in Phys. Res. A572, 526 (2007).

[7] Greg Derylo, H. Thomas Diehl, Juan Estrada, “0.25 mm-Thick CCD Packaging for the Dark Energy Survey Camera Array", High Energy, Optical, and Infrared Detectors for Astronomy II. Edited by Dorn, David A.; Holland, Andrew D., Proceedings of the SPIE, Volume 6276, 627608 (2006). This paper describes the previous version of the CCD package. A key modification in the present design is that the glue gap between the CCD and AlN blank is held by the assembly hardware, rather than by tiny squares of 4-mil thick double-stick tape.

[8] G. Derylo et al., "Surface cleaning of CCD imagers using an electrostatic dissipative formulation of First Contact $^{T M}$, , these proceedings.

[9] “CCD 42-90 Back Illuminated Deep Depletion 2048 x 4068 Pixel Scientific CCD Sensor”, http://www.e2v.com.

[10] H. Cease et al., "Measurements of Charge Diffusion in Deep-Depletion CCDs by Optical Diffraction", Experimental Astronomy, (2007). At this time the article is only available online at http://www.SpringerLink.com.

[11] Jessamyn A. Fairfield et al., "Improved spatial resolution in thick, fully depleted CCDs with enhanced red sensitivity”, IEEE Trans. Nucl. Sci. 53 (6), 3877-3881 (2006).

[12] H. Cease et al., "The Dark Energy Survey CCD Imager Design", these proceedings.

[13] R. L. Schmitt et al., "Cooling the Dark Energy Camera Instrument”, these proceedings. 